\title{
Partizipation in der Zusammenarbeit zwischen Zielgruppe, Projekt und Geldgeber
}

\section{Participation in the Cooperation between Target Group, Project and Sponsor}

\author{
Autoren \\ M. T. Wright, M. Block, H. von Unger \\ Institut \\ Wissenschaftszentrum Berlin für Sozialforschung (WZB)
}

\author{
Schlüsselwörter \\ - partizipative \\ Qualitätsentwicklung \\ - partizipative \\ Gesundheitsforschung \\ - lebensweltorientierte \\ Gesundheitsförderung \\ - Zusammenarbeit \\ - Partizipation \\ - Empowerment \\ Key words \\ - community-based \\ participatory research \\ - health promotion \\ - quality assurance \\ - decision-making \\ - power \\ - stakeholders
}

\section{Zusammenfassung \\ $\checkmark$}

Die intrinsische Verbindung von Empowerment und Partizipation wird in der Ottawa-Charta zur Gesundheitsförderung deutlich: Um Autonomie und Kontrolle über die eigenen Gesundheitsbelange zu erlangen (Empowerment), müssen Bürger die Möglichkeit haben, einen Einfluss auf ihre Umwelt zu nehmen (Partizipation). Dies impliziert, dass die Entscheidungsmacht über wesentliche Faktoren, die mit dem Gesundheitszustand zusammenhängen, oft nicht in den Händen der Bürger liegt. Es stellt sich die Frage: Wie kann diese Macht so (um)verteilt werden, dass Bürger mehr selbst bestimmen können? Diese Frage ist nicht nur auf höchster politischer Ebene relevant, sondern auch im Rahmen der konkreten Zusammenarbeit zwischen lokalen Akteuren, denn hier werden wesentliche Entscheidungen über die Ausrichtung und Ausstattung gesundheitsfördernder Maßnahmen getroffen. Vor Ort kann ein zentraler Beitrag zur Stärkung von Nachbarschaften und Gemeinden und damit zu lebensweltorientierter Gesundheitsförderung realisiert werden. In diesem Artikel wird die Methode „Kreise der Entscheidung“ vorgestellt, die dazu dient, den Grad der erreichten Partizipation verschiedener Akteure an der Gesundheitsförderung zu überprüfen. Diese Methode basiert auf dem Ansatz der Partizipativen Qualitätsentwicklung, der von den Autoren und ihren Praxispartnern konzipiert wurde. Auf der Grundlage eines gemeinsamen Verständnisses von Partizipation als Entwicklungsprozess wird der Aufbau partizipativer Formen der Zusammenarbeit erleichtert - zum einen, weil die verschiedenen Ausprägungen der Partizipation konkretisiert werden, und zum anderen, weil die Möglichkeit zur Überprüfung der eigenen Zusammenarbeit nach dem aktuellen und erwünschten Stand der Partizipation besteht. Dadurch wird die Diskus-

\section{Abstract \\ $\nabla$}

The intrinsic connection between empowerment and participation is apparent in the Ottawa Charter on Health Promotion. In order for citizens to reach a higher degree of autonomy and control over health-related factors (empowerment) they need to have an active role in the decision-making processes affecting their lives and the environment in which they live (participation). This implies that many decisions are made affecting the health of citizens over which they have no influence. The question is: Who has the power to make such decisions and how can this power be shared more equitably? This question can be raised not only at the highest political level, but also locally in the context of the collaboration between various stake-holders. The local level plays a key role in deciding which health promotion measures are developed and funded, thus contributing in an important way to strengthening communities. In this article the method "Circles of Decision-Making" is presented as a tool for assisting those working at the local level in determining to what degree the active participation of the various stake-holders has been achieved and in what ways the participation of those "on the outside" of decision-making processes can be strengthened. This method is based on the concept of Participatory Quality Development (PQD) created by the authors and their community partners. PQD uses methods from community-based research to address issues of quality in community-level health promotion and prevention. 
sion über Partizipation transparenter und für die Beteiligten handhabbar.

\section{Empowerment, Partizipation und Zusammenarbeit in der Gesundheitsförderung}

In der Ottawa-Charta [1] der WHO werden Empowerment und Partizipation als integrierte Bestandteile der Gesundheitsförderung erklärt. Der in der englischen Fassung des Textes verwendete Begriff „empowerment“ wird durch das deutsche Wort „Selbstbestimmung“ und „participation“ durch „Teilnahme und Mitbestimmung“ übersetzt:

„Gesundheitsförderung wird realisiert im Rahmen konkreter und wirksamer Aktivitäten von Bürgern in ihrer Gemeinde: In der Erarbeitung von Prioritäten, der Herbeiführung von Entscheidungen sowie bei der Planung und Umsetzung von Strategien. Die Unterstützung von Nachbarschaften und Gemeinden im Sinne einer vermehrten Selbstbestimmung ist ein zentraler Angelpunkt der Gesundheitsförderung; ihre Autonomie und Kontrolle über die eigenen Gesundheitsbelange ist zu stärken.

Die Stärkung von Nachbarschaften und Gemeinden baut auf den vorhandenen menschlichen und materiellen Möglichkeiten der größeren öffentlichen Teilnahme und Mitbestimmung auf. Selbsthilfe und soziale Unterstützung sowie flexible Möglichkeiten der größeren öffentlichen Teilnahme und Mitbestimmung für Gesundheitsbelange sind dabei zu unterstützen bzw. neu zu entwickeln. Kontinuierlicher Zugang zu allen Informationen, die Schaffung von gesundheitsorientierten Lernmöglichkeiten sowie angemessene finanzielle Unterstützung gemeinschaftlicher Initiativen sind dazu notwendige Voraussetzungen“.

Die intrinsische Verbindung von Empowerment und Partizipation wird im Text deutlich: Um „Autonomie und Kontrolle über die eigenen Gesundheitsbelange“ zu erlangen (Empowerment), müssen Bürger ${ }^{1}$ die Möglichkeit haben, einen Einfluss auf ihre Umwelt zu nehmen (Partizipation). Diese Forderung impliziert, dass die Entscheidungsmacht über wesentliche Faktoren, die mit dem Gesundheitszustand zusammenhängen, oft nicht in den Händen der Bürger liegt. Es stellt sich die Frage: Wer hat diese Macht und wie kann sie so verteilt werden, dass Bürger mehr selbst bestimmen können?

Diese Frage ist nicht nur auf höchster politischer Ebene, sondern auch im Rahmen der konkreten Zusammenarbeit zwischen Akteuren vor Ort relevant, denn hier werden wesentliche Entscheidungen über die Ausrichtung und Ausstattung gesundheitsfördernder Maßnahmen getroffen, und hier kann ein zentraler Beitrag zur „Stärkung von Nachbarschaften und Gemeinden“ realisiert werden.

Sowohl international als auch in Deutschland hat in den letzten Jahren die Bedeutung von Zusammenarbeit zwischen Akteuren bei der Umsetzung der (lebensweltorientierten) Gesundheitsförderung und Prävention zugenommen. Dieser Entwicklung liegt die Erkenntnis zugrunde, dass nicht isolierte Einzelmaßnahmen, sondern koordinierte, flächendeckende, vielschichtige Strategien die beste Chance haben, die Gesundheitslage sozial benachteiligter Bevölkerungsgruppen nachhaltig zu verbessern [2] Der Kooperationsverbund zur Gesundheitsförderung bei sozial Benachteiligten auf Bundesebene [3] sowie integrierte Handlungskonzepte auf Stadteilebene [4] sind zwei bedeutende

\footnotetext{
${ }^{1}$ Im Interesse der Lesbarkeit wird ausschließlich die männliche Form ver-
} wendet. Gemeint sind weibliche und männliche Personen.
Beispiele dieser Entwicklung. Auf kommunaler und regionaler Ebene bauen die neuen Bündnisse nicht selten auf bereits existierenden Arbeitskreisen und Netzwerken auf, die sich seit längerer Zeit um die Stärkung der Zusammenarbeit zwischen diversen Akteuren des Sozial- und Gesundheitswesens bemühen.

\section{Der Ansatz der Partizipativen Qualitätsentwicklung $\nabla$}

Empowerment, Partizipation und Zusammenarbeit sind zentrale Merkmale des von uns und unseren Praxispartnern entwickelten Ansatzes der Partizipativen Qualitätsentwicklung, der auf eine möglichst starke Teilnahme und Teilhabe (Partizipation) der Projektmitarbeiter und vor allem der Zielgruppen an allen Aspekten der Planung, Durchführung, Steuerung und Auswertung präventiver und gesundheitsfördernder Maßnahmen abzielt. Die Partizipative Qualitätsentwicklung soll Projekte befähigen, in einer gleichberechtigten Zusammenarbeit mit Zielgruppe, Geldgeber und eventuell anderen wichtigen Akteuren kontinuierlich relevante Daten über ihre eigene Arbeit zu erheben und als Grundlage für die ständige Verbesserung der geleisteten Maßnahmen (Qualitätsentwicklung) einzusetzen. Durch ihre Partizipation an der Gesundheitsförderung und Qualitätsentwicklung werden Menschen aus der Zielgruppe in die Lage versetzt, Interventionen mitzuentwickeln, die ihre Lebenswelten zugunsten ihrer Gesundheit verändern (Empowerment). Auch Projektmitarbeiter entwickeln neue Kompetenzen, bzw. entwickeln bereits bestehende Kompetenzen weiter, um ihren Praxiszusammenhang positiv zu beeinflussen. Dabei ist die Rolle der Wissenschaft im Wesentlichen begleitend: Sie stellt vor allem ihre Methodenkompetenzen hinsichtlich der Erhebung und Interpretation von Daten zur Verfügung. Partizipative Qualitätsentwicklung steht in der Tradition der community-based participatory research, die vor allem im angloamerikanischen Raum, in Skandinavien sowie in der Entwicklungsarbeit weit verbreitet ist [5-7].

Die kleinste Einheit der Zusammenarbeit, die auf jeden Fall zustande kommen muss, um die Umsetzung der Partizipativen Qualitätsentwicklung zu ermöglichen, ist diejenige zwischen Zielgruppe, Projekt und Geldgeber. In diesem „Beziehungsdreieck" werden spezifische Maßnahmen konzipiert und umgesetzt. Ausgehend von einem Anliegen des Geldgebers und/oder eines Projektträgers, kommen Geldgeber und Projektträger ins Gespräch, um sich über eine angemessene Intervention zu verständigen, die der Zielgruppe gesundheitlich zugute kommen soll. Seltener sind es Vertreter aus der Zielgruppe selbst, die die Gespräche mit einem Träger oder einem Geldgeber initiieren, um neue gesundheitsfördernde bzw. präventive Angebote zu realisieren. Unabhängig davon, wer die Initiative ergreift, wird bereits bei der ersten Phase der Zusammenarbeit (Definition des Problems, Zielsetzung) eine (Arbeits-)Beziehung aufgebaut, in der Geldgeber, Projektträger und Zielgruppe aufeinander angewiesen sind. Der Geldgeber kann die Zielgruppe nur durch die Arbeit des Projekts erreichen. Das Projekt kann nur mit der Förderung des Geldgebers die Maßnahme umsetzen. Und die Zielgruppe bekommt die vorgesehene Hilfe erst, wenn der Geldgeber und der Projektträger ein Angebot realisieren. Wenn einer der drei Beteiligten sich aus dem Dreieck zurückzieht oder sich nicht oder nur begrenzt auf eine Beziehung einlässt, kommt keine erfolgreiche Intervention zustande. Fehlende oder nicht ausreichende Fördermittel (Geldgeber), ein nicht existierendes oder unangemessenes Angebot (Projektträger) und eine schwache 
Akzeptanz oder fehlende Inanspruchnahme des Angebots (Zielgruppe) sind Folgen einer misslungenen oder unvollständigen Zusammenarbeit.

Die Partizipative Qualitätsentwicklung will durch den Aufbau einer Kooperation zwischen den Beteiligten eine Situation schaffen, in der die oft divergierenden Interessen zusammengeführt und Lösungen ausgehandelt werden können. Hierfür muss die Partizipation aller Beteiligten (im Sinne von Einflussnahme auf Entscheidungsprozesse, s. u.) gewährleistet werden. Die vorhandene Hierarchie unter den Beteiligten, die durch Macht- und Ressourcengefälle bedingt ist, kann jedoch eine erhebliche Barriere für die Realisierung der Partizipation darstellen. Die Entscheidungsmacht, ob eine Maßnahme überhaupt initiiert wird, liegt beim Geldgeber: Er verfügt unmittelbar über die Fördermittel und trägt oft die alleinige Verantwortung für eine ordnungsgemäße Vergabe der Mittel. Diese Position stützt sich u.a. auf Richtlinien, institutionell festgelegte Entscheidungsbefugnisse und den beruflichen Status der zuständigen Mitarbeiter. Am anderen Ende stehen sozial benachteiligte Bevölkerungsgruppen, die definitionsgemäß über begrenzte Ressourcen sowie einen niedrigen sozialen Status verfügen und in den meisten Fällen keine institutionalisierte Vertretung oder verbindliche Einflussnahme auf die Gestaltung von Maßnahmen haben. Die Bezeichnung „Zielgruppe“ ist ohnehin ein Konstrukt von Praktikern und Geldgebern, das auf wissenschaftlichen bzw. von der Praxis definierten Kriterien des Bedarfs fußt und selten der Selbstdefinition der Menschen entspricht, die erreicht werden sollen. Zwischen dem Geldgeber und der Zielgruppe agiert der Projektträger, dem durch seine praktischen Kompetenzen und/ oder fachliche Qualifikation ein gewisses Mitspracherecht hinsichtlich der Gestaltung von Interventionen durch Geldgeber zugestanden wird. Wegen dieses Macht- und Ressourcengefälles und der damit verbundenen eingeschränkten Einflussnahme auf Entscheidungen über die Förderung von Maßnahmen betont die Partizipative Qualitätsentwicklung die Stärkung der Partizipation der Projekte und vor allem der Zielgruppen. Dadurch sollen die Interessen der Projektmitarbeiter und Zielgruppenmitglieder besser artikuliert und berücksichtigt werden, um die Konzeption und Durchführung von passgenauen und zielgruppenspezifischen Interventionen zu ermöglichen.

\section{Zur Bestimmung des Ausmaßes der Partizipation in der Zusammenarbeit}

Der Begriff der „Partizipation“ bedarf der Definition. Es ist insbesondere notwendig, verschiedene Formen von Beteiligung auch im Hinblick auf das Ausmaß von Mitbestimmung und Entscheidungsmacht zu differenzieren. Im Rahmen der Partizipativen Qualitätsentwicklung bedeutet Partizipation Entscheidungsmacht: Je größer die Entscheidungsmacht einer Person oder einer Gruppe, umso größer ist der Grad an Partizipation. Diese Definition gründet auf der zentralen Forderung der OttawaCharta, Selbstbestimmung der Bürger als Kern der Gesundheitsförderung zu realisieren. Sie basiert zudem auf einer langjährigen Diskussion in der Stadtentwicklung und später in der Entwicklungsarbeit über die Rolle von Bürgern in der Realisierung von Maßnahmen, die ihre Umgebung verbessern sollen. Diese Diskussion wurde maßgeblich von der Arbeit der US-Amerikanerin Sherry Arnstein [8] beeinflusst, die bereits Ende der 60er Jahre die Gründe für erfolgreiche Bürgerinitiativen analysierte. Sie schlussfolgert, dass Veränderungen in Wohnvierteln, die den Alltag der Anwohner nachhaltig verbessern, erst dann verwirklicht werden, wenn die Bürger ihre Lebensbedingungen (mit-)be- stimmen können. In ihrem Aufsatz zieht Arnstein eine klare Grenze zwischen „echter“ Partizipation auf der einen Seite und „Scheinpartizipation“ auf der anderen. Sie stellt dabei fest, dass die meisten partizipativ angelegten Prozesse keine wirkliche Partizipation zulassen. Um die verschiedenen Ausprägungen der Partizipation zu beschreiben, schlägt Arnstein ein Stufenmodell vor („Ladder of Participation“).

Unter Berücksichtigung der Ergebnisse unserer Forschungstätigkeiten und mit Bezug auf andere Arbeiten in Anlehnung an Arnstein $[9,10]$, haben wir ein Modell entwickelt, das ermöglichen soll, existierende und erwünschte partizipative Prozesse auf der Projektebene näher zu beschreiben. In dem Modell zu „Stufen der Partizipation in der Gesundheitsförderung und Prävention“ [11] werden Projektanbietern Anhaltspunkte geboten, um den Grad der in ihrer Arbeit erreichten Partizipation einzuschätzen und Möglichkeiten zu ihrer Steigerung zu entwickeln. Es kann u. a. einsetzt werden, um den Grad der Partizipation in der Zusammenarbeit zwischen Akteuren auf der lokalen Ebene zu beschreiben. Für diese spezifische Anwendung des Modells haben wir zusammen mit unseren Praxispartnern die Methode „Kreise der Entscheidung“ konzipiert.

\section{Die „Kreise der Entscheidung“ als Instrument der Partizipativen Qualitätsentwicklung}

Durch die „Kreise der Entscheidung“ kann die Einflussnahme der verschiedenen Akteure (Projektmitarbeiter, Zielgruppe, Zuwendungsgeber und evtl. weitere Kooperationspartner) auf Entscheidungen zur Gesundheitsförderung und Prävention bildlich dargestellt werden, auch im Verhältnis zu einander. Auf der Grundlage dieser Darstellung können Strategien zur Stärkung der Partizipation solcher Akteure entwickelt werden, die bisher nur schwach an Entscheidungsprozessen beteiligt sind. Mit Hilfe der „Kreise der Entscheidung“ kann geklärt werden - oft zum ersten Mal -, wie Entscheidungsprozesse verlaufen und wer an welchen Entscheidungen beteiligt ist: Entscheidungsmacht kann erst geteilt werden, wenn Klarheit darüber herrscht, wer sie ausübt! So lässt sich reflektieren, wie es aktuell um die Partizipation in der Zusammenarbeit steht (Ist-Zustand) und wie eine zukünftige Zusammenarbeit partizipativer gestaltet werden kann (Soll-Zustand).

Für die „Kreise der Entscheidung“ wird ein Kreisdiagramm verwendet, das ein Bild über die relative Einflussnahme der verschiedenen Akteure auf Entscheidungen zeichnet (s. $\bullet$ Abb. 1): Es lässt sich ablesen, welcher Akteur anteilig wie viel Entscheidungsmacht hat. Jeder Kreis entspricht einer der Stufen aus den Kategorien „Vorstufen der Partizipation“ bzw. „Partizipation“. Die zusätzliche Kategorie „nicht beteiligt“ umfasst diejenigen, die aktuell nicht an der Zusammenarbeit teilnehmen. Je weiter ein Akteur vom inneren Kreis entfernt ist, umso weniger Partizipation (Teilhabe an Entscheidungsprozessen) wird realisiert.

Bei den Vorstufen der Partizipation werden die Akteure zunehmend stark in Entscheidungsprozesse eingebunden, auch wenn (noch) kein direkter Einfluss auf diese Prozesse möglich ist, so durch Information, Anhörung oder Einbeziehung. Bei der „echten“ Partizipation haben die Akteure eine formale, verbindliche Rolle in der Entscheidungsfindung (s. $\bullet$ Tab. 1).

Nach unserem Verständnis ist Partizipation kein Entweder/Oder, sondern ein Entwicklungsprozess. In vielen Settings müssen zunächst Vorstufen der Partizipation realisiert werden, bevor eine direkte Beteiligung an Entscheidungsprozessen möglich ist. Allerdings muss die Anmahnung von Arnstein ernst genommen werden: Viele Maßnahmen, die sich für partizipativ halten, bie- 


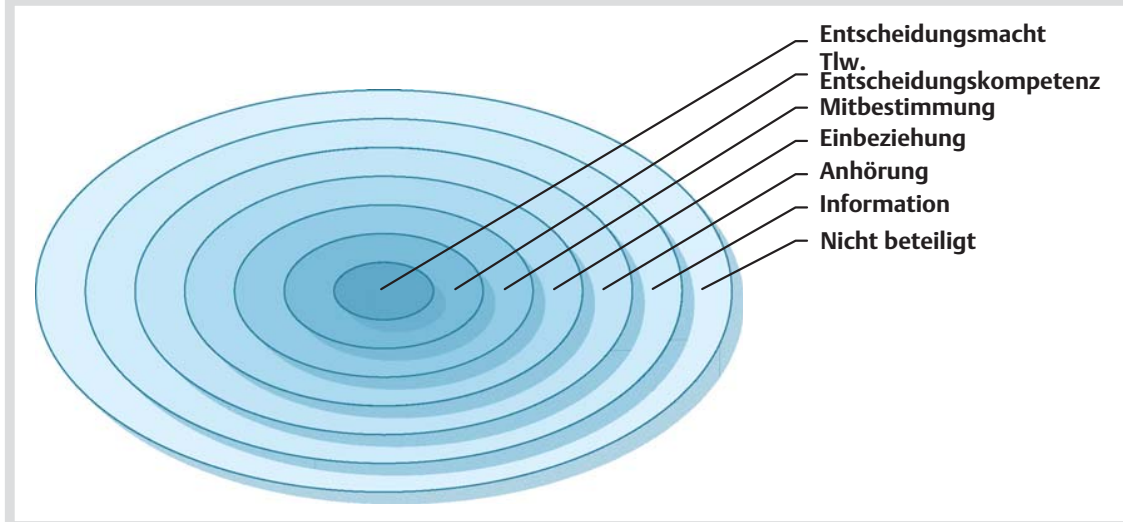

Abb. 1 Kreise der Entscheidung.

Tab. 1 Kategorien der Partizipation im Rahmen der „Kreise der Entscheidung“.

\begin{tabular}{|c|c|}
\hline \multicolumn{2}{|l|}{ Vorstufen der Partizipation } \\
\hline Information & $\begin{array}{l}\text { In diesem Kreis sind Personen, die von } \\
\text { den Entscheidungsträgern über die (ge- } \\
\text { plante) Maßnahme informiert werden. } \\
\text { Rückmeldungen von diesen Personen } \\
\text { werden weder gesucht noch erwartet. }\end{array}$ \\
\hline Anhörung & $\begin{array}{l}\text { Personen in diesem Kreis werden bei } \\
\text { Bedarf von den Entscheidungsträgern } \\
\text { konsultiert. Der Kontakt zwischen } \\
\text { diesen Personen und den Entschei- } \\
\text { dungsträgern ist unverbindlich und } \\
\text { üblicherweise unregelmäßig. }\end{array}$ \\
\hline Einbeziehung & $\begin{array}{l}\text { Die Entscheidungsträger lassen sich von } \\
\text { den Personen in diesem Kreis beraten. } \\
\text { Die Beratungen haben jedoch keinen } \\
\text { verbindlichen Einfluss auf den Entschei- } \\
\text { dungsprozess. }\end{array}$ \\
\hline \multicolumn{2}{|l|}{ „Echte“ Partizipation: } \\
\hline Mitbestimmung & $\begin{array}{l}\text { Personen in diesem Kreis wird ein } \\
\text { Mitspracherecht zugeteilt. Zu wesent- } \\
\text { lichen Aspekten der Maßnahme halten } \\
\text { die Entscheidungsträger mit ihnen } \\
\text { Rücksprache. Diese Personen haben } \\
\text { jedoch keine alleinigen Entscheidungs- } \\
\text { befugnisse. }\end{array}$ \\
\hline $\begin{array}{l}\text { teilweise Entscheidungskom- } \\
\text { petenz }\end{array}$ & $\begin{array}{l}\text { Personen in diesem Kreis können } \\
\text { über bestimmte, klar eingeschränkte } \\
\text { Aspekte der Maßnahme selbst ent- } \\
\text { scheiden. }\end{array}$ \\
\hline Entscheidungsmacht & $\begin{array}{l}\text { In diesem Kreis befinden sich die } \\
\text { Akteure, die an allen wichtigen } \\
\text { Entscheidungen direkt beteiligt sind. } \\
\text { Diese Personen bestimmen alle Aspekte } \\
\text { der Maßnahme (Problemdefinition, } \\
\text { Planung, Durchführung, Auswertung). }\end{array}$ \\
\hline
\end{tabular}

ten keine Möglichkeit für eine Beeinflussung der Entscheidungsprozesse durch die Bürger und sind daher nicht als partizipativ einzustufen. In Arnsteins Worten: „Es gibt einen wesentlichen Unterschied zwischen dem Durchlaufen eines leeren Rituals der Partizipation und dem Verfügen über die Macht, die man braucht, um die Ergebnisse des Prozesses zu beeinflussen [8]“.

\section{Zur Anwendung der „Kreise der Entscheidung“}

Zuerst wird von dem Projektmitarbeiter der Gegenstand der Untersuchung ausgewählt. Eine Einrichtung, ein Arbeitsbereich, ein spezifisches Projekt oder eine einzelne Intervention können hier in Frage kommen. Dann ist zu entscheiden, welche Akteurskonstellation für den Erfolg der gesundheitsfördernden bzw. präventiven Arbeit erforderlich ist. Hierbei beschränken sich Praktiker üblicherweise nicht auf das oben genannte „Beziehungsdreieck“ (Zielgruppe, Projekt, Geldgeber), sondern schließen andere (potenzielle) Kooperationspartner ein (Politiker, Stiftungen, Ämter, Krankenkassen, andere Träger usw.). Anschließend wird eine Liste der Akteure erstellt (Einrichtungen, Gruppen sowie - wo vorhanden und relevant - einzelne Personen aus den Einrichtungen und Gruppen), deren Partizipation von Bedeutung ist. Die Akteure werden in einem nächsten Schritt den einzelnen Kreisen des Diagramms zugeordnet. In den innersten Kreis kommen die Akteure, die aktuell Entscheidungen für das Projekt treffen. Je weiter ein Akteur vom Zentrum entfernt ist, umso geringer ist sein Einfluss auf die Entscheidungsfindung. Die oben aufgeführten Bezeichnungen der einzelnen Kreise und deren Beschreibungen helfen dem Praktiker, die Akteure zuzuordnen. Auf diese Weise entsteht ein Bild des Ist-Zustands der anteiligen Entscheidungsmacht (Partizipation) der Akteure.

Spätestens bei der Zuordnung der Akteure erkennt der Praktiker, ob er weiß, wie Entscheidungen im Projekt getroffen werden. Die Konzipierung, Durchführung und Auswertung von Interventionen im Bereich der Gesundheitsförderung und Prävention erfolgen oft nicht im Rahmen eines klar strukturierten Verfahrens. Wer wann welche (Entscheidungs-)Befugnisse hat, muss für die Übung häufig rekonstruiert werden. Ein wichtiges Zwischenergebnis ist für viele Praktiker die Analyse und Reflexion, wie die formellen und informellen Entscheidungsprozesse verlaufen und wer Einfluss auf diese Prozesse nimmt. Diese Erkenntnis ist eine Voraussetzung für die Stärkung der Partizipation.

Im nächsten Schritt wird der Soll-Zustand skizziert: Welche Akteure sollen an Entscheidungsprozessen in Zukunft beteiligt werden und wie? Hierfür wird ein zweites Kreisdiagramm erstellt.

Die letzte Phase der Übung dient der Lösungsfindung, wie die Partizipation der einzelnen Akteure gestärkt werden kann: Im Vordergrund steht die Frage, was der Praktiker oder seine Einrichtung/Gruppe tun kann oder muss, um den Soll-Zustand zu realisieren.

Die Kreise der Entscheidung können durch Methoden aus dem Psychodrama im Rahmen einer Aufstellung interaktiv als Gruppenprozess gestaltet werden, zum Beispiel auf einem Workshop mit Vertretern aus verschiedenen Einrichtungen (wie im Rahmen unserer Forschungsprojekte) oder in einem Mitarbeiterteam einer einzelnen Einrichtung [12]. Hierbei wird zunächst von jedem Teilnehmer der Ist-Zustand in dem von ihm ausge- 
wählten Bereich auf Papier skizziert. Unterstützt durch die Moderation stellt anschließend ein Teilnehmer sein Bild räumlich vor, indem er die Kreise auf dem Boden markiert. Zuerst lokalisiert der Teilnehmer seine Position in den „Kreisen der Entscheidung“ (Wie nah oder fern ist er selbst dem Zentrum der Entscheidungsmacht?). Dann wählt der Teilnehmer für jeden Akteur eine Person aus der Gruppe, die den Akteur symbolisiert. Der „stellvertretende Akteur“ bezieht in den ihm zugewiesenen Kreis Position. Der agierende Teilnehmer ordnet den „stellvertretenden Akteuren“ jeweils einen Spruch sowie eine Körperhaltung oder Geste zu, die die Einstellung des dargestellten Akteurs zum Ausdruck bringen. Spruch, Körperhaltung und Geste werden geübt, bis der Teilnehmer mit der Darbietung zufrieden ist. Nachdem auf diese Weise alle Akteure aufgestellt wurden, spielt nacheinander (die Reihenfolge wird im Vorfeld bestimmt) jeder Darsteller „seine Rolle“. Dies kann zwei oder drei Mal wiederholt werden. Nun wird der Teilnehmer nach seinen Empfindungen und Gedanken gefragt, die die Aufstellung auslöst hat. Der Fokus liegt dabei auf neuen Erkenntnissen und Ideen. Es geht darum, neue Impulse für die Reflexion der Zusammenarbeit zu erzeugen. Anschließend geben die Darsteller Feedback zu ihren Rollen und zum Gesamtbild. Die hier geäußerten kritischen Fragen und Anmerkungen der Darsteller können den Teilnehmer anregen, seine Vorstellungen der Zusammenarbeit zu überdenken. In einem nächsten Schritt werden die in der Aufstellung nicht integrierten Gruppenmitglieder um Rückmeldung gebeten.

In der letzten Phase der interaktiven Übung wird der Soll-Zustand dargestellt. Der Teilnehmer erhält einen „Zauberstab“, um die dargestellten Akteure zu verwandeln. Nun kann sich alles ändern - sowohl der Kreis, in dem der Akteur steht, als auch sein Spruch, seine Köperhaltung und Gestik. Auch der Teilnehmer selbst kann seine Position innerhalb der Kreise neu bestimmen. Der Zauberstab hebt den Realitätsbezug auf - alles ist möglich. Der so geschaffene Freiraum eröffnet bisher nicht wahrgenommene oder „erträumte“ Möglichkeiten, aus denen sich Anregungen für den Umgang mit den Akteuren in der „realen“ (Berufs-)Welt ableiten lassen.

\section{Wann ist die optimale Partizipation aller Akteure erreicht?}

Die Praktiker, mit denen wir im Rahmen unserer Forschungsprojekte zur Partizipativen Qualitätsentwicklung zusammenarbeiten, lehnen eine ständige Positionierung aller Akteure im engeren Kreis der Entscheidungsmacht ab. Dem häufig in der Literatur vertretenen Ansatz der Gleichberechtigung aller Beteiligten stehen die Praktiker kritisch gegenüber. So gewinnen im Soll-Zustand manche Akteure an Einfluss, während andere ihren Platz behalten oder an Einfluss verlieren. Dies wird häufig durch Argumente der Autonomie und der Fachlichkeit, gelegentlich durch Zeitmangel und einen möglichen hohen Aufwand begründet. Nicht jeder Akteur kann und will an jeder Entscheidung, die getroffen werden muss, beteiligt sein. Die Projektmitarbeiter möchten Fördermittel für ihre Arbeit bekommen, aber mit so wenig Kontrolle und Vorgaben seitens des Geldgebers wie möglich, um ihre Autonomie zu wahren. Der Zielgruppe wird zum Teil mehr Mitspracherecht als dem Geldgeber eingeräumt, aber auch die Zielgruppe steht nur selten im „inneren“ Kreis der Entscheidungsmacht. Am häufigsten erscheint eine Konstellation von Praktikern aus der gleichen Einrichtung im inneren Kreis, die alle Aspekte einer Maßnahme bestimmen. Durch diese Autonomie der Praktiker soll eine Fachlichkeit gewährleistet werden, über die weder die Zielgruppe noch der Geldgeber verfügen: Projektentscheidungen sollen von den im Projekt angestellten Fachkräften getroffen und müssen auch von diesen verantwortet werden.

Andere Sichtweisen auf Entscheidungsprozesse entstehen, wenn im Fall einer spezifischen Intervention nicht die Projektmitarbeiter, sondern Vertreter der Zielgruppe oder zuständige Mitarbeiter des Geldgebers die „Kreise der Entscheidung“ einsetzen, um den Ist- und Soll-Zustand einer Maßnahme der Prävention oder Gesundheitsförderung zu beschreiben. Hier erhält die Zielgruppe bzw. der Geldgeber mehr Entscheidungskompetenz, und auch die relative Einflussnahme der übrigen Akteure wird anders verteilt.

Wie auch andere Instrumente der Partizipativen Qualitätsentwicklung zielt die Methode der „Kreise der Entscheidung“ darauf, die Zusammenarbeit zwischen Akteuren zu stärken. Hierzu wird die Vielfalt an Perspektiven auf die Partizipation an Entscheidungsprozessen vor Ort transparent gemacht, damit die lokalen Akteure Möglichkeiten der Weiterentwicklung der Zusammenarbeit offen aushandeln können. Qualitätsentwicklung ist ein Diskurs, der vom Austausch unter den Beteiligten lebt und der durch partizipative Verfahren gefördert wird $[13,14]$. Entsprechend dem jeweiligen Umfeld wird sowohl die Akteurskonstellation als auch die den einzelnen Akteuren zugeordnete Entscheidungskompetenz variieren. Die Partizipative Qualitätsentwicklung setzt jedoch voraus, dass sich alle Beteiligten um eine kritische Reflexion der aktuellen Partizipation bemühen. Es gilt zu analysieren, inwieweit Entscheidungsmacht geteilt wird, und zu ermöglichen, dass vor allem die Entscheidungsmacht der Zielgruppe gestärkt wird.

\section{Einflussfaktoren auf die Realisierung von Partizipa- tion \\ $\nabla$}

Zahlreiche Faktoren beeinflussen, ob Partizipation in der $\mathrm{Zu}$ sammenarbeit zwischen Projekt, Zielgruppe, Geldgeber und anderen Akteuren realisiert werden kann. Im Rahmen unserer Forschungsarbeit wurden zwei Gruppen von Praktikern gebeten, die aus ihrer Erfahrung wichtigsten Faktoren zu erörtern. Die folgende Darstellung gibt die Meinung der befragten Praktiker wieder.

Als Ergebnis der Gruppengespräche konnten folgende Aspekte herausgearbeitet werden:

- Haltung,

- Aufwand,

- Pflege der Zusammenarbeit,

- Interessen,

- Einigkeit in der eigenen Einrichtung und

- Fachlichkeit.

Bei jedem Aspekt wurde weiter nach dem Kooperationspartner unterschieden: Zielgruppe, Geldgeber und anderen Kooperationspartnern.

\section{Haltung}

Die Partizipation der Zielgruppe verlangt u.U. einen Perspektivwechsel in der Arbeit: Es handelt sich nicht (mehr) in erster Linie darum, ein Projekt für, sondern mit der Zielgruppe durchzuführen. Die Entwicklung der Partizipation ist ein Prozess, auf den sich ein Projekt einlassen muss. Dazu gehören Vertrauen zu der Zielgruppe, Geduld im Prozess und Disziplin bei der Durchführung der Bemühungen, Partizipation zu stärken. Aufgrund 
des Misstrauens, das manche sozial benachteiligten Menschen gegenüber Einrichtungen der Sozialen Arbeit empfinden, kann der Aufbau einer vertrauensvollen Zusammenarbeit zwischen Projekt und Zielgruppe problematisch sein. Ein Hindernis aus Sicht vieler Einrichtungen, die der Zielgruppe eine stärkere Partizipation ermöglichen wollen, ist der Mangel an Interesse seitens des Geldgebers, die Partizipation der Zielgruppe als Projektziel explizit zu unterstützen.

Eine stärkere Partizipation von Projektmitarbeitern an Entscheidungsprozessen verlangt häufig eine Anpassung an die Erwartungen des Geldgebers, um verstanden und ernst genommen zu werden. Der Grad der notwendigen Anpassung ist unterschiedlich. Im Allgemeinen halten Praktiker es für wichtig, in der Zusammenarbeit mit Geldgebern eine authentische Haltung zu wahren; sich so zu geben, wie man im (Praxis-)Alltag ist, kann die Glaubwürdigkeit des Auftritts steigern. Eine weitere Voraussetzung für eine stärkere Partizipation eines Projekts an Entscheidungen hinsichtlich geförderter Maßnahmen ist das Vertrauen durch den Geldgeber. Eine überhöhte Anspruchshaltung seitens des Projekts kann eine partizipative Zusammenarbeit behindern.

In der Zusammenarbeit mit anderen Kooperationspartnern müssen Projektmitarbeiter akzeptieren, dass einige Einrichtungen aufgrund ihres Konzepts oder ihrer (hierarchischen) Struktur Entscheidungskompetenzen nicht teilen dürfen. Konkurrenz zwischen Einrichtungen ist ein weiterer Grund, warum partizipative Formen der Zusammenarbeit nicht zustande kommen.

\section{Aufwand}

Der Aufbau einer Zusammenarbeit - ob mit der Zielgruppe oder anderen Kooperationspartnern - ist ein aufwändiger Prozess, der erst über einen längeren Zeitraum und durch die Investition von Ressourcen (Personal- und Sachmittel) initiiert und entwickelt werden kann. Kurzfristig betrachtet können deshalb partizipative Formen der Zusammenarbeit kostenintensiver als nicht partizipative Formen sein. Längerfristig werden jedoch die Angebote der Gesundheitsförderung und Prävention, die durch partizipative Prozesse entwickelt und umgesetzt wurden, wirkungsvoller sein, weil sie durch Einbindung der Zielgruppe besser in die Lebenswelt sozial benachteiligter Menschen integriert werden können.

\section{Pflege der Zusammenarbeit}

Für alle Kooperationspartner gilt, dass die Zusammenarbeit nicht von selbst entsteht, sondern von allen Beteiligten aufgebaut und gepflegt werden muss.

In der Zusammenarbeit mit der Zielgruppe bedeutet dies eine Steuerung (z.B. in Form einer strategischen Planung zur Stärkung der Zusammenarbeit), um mit der Zeit eine größere Partizipation der Zielgruppe zu ermöglichen. Konsumverhalten der Zielgruppe kann ein Hindernis beim Aufbau partizipativer Strukturen darstellen. Vor diesem Hintergrund können Konfrontationen zwischen Projektmitarbeitern und Mitgliedern der Zielgruppe notwendig sein, um die Zielgruppe zu motivieren, eine passive Empfänger-Haltung aufzugeben und eine aktive Teilhabe am Projektgeschehen zu entwickeln. Hierbei kann es hilfreich sein, der Zielgruppe deutlich zu machen, wie wichtig ihr Engagement für die Aufrechterhaltung der Einrichtung ist.

Die Form der Zusammenarbeit mit Geldgebern sollte mit Sorgfalt bestimmt werden. Eine Partnerschaft im Sinne einer Gleichberechtigung im Hinblick auf alle Projektentscheidungen ist für die Projektmitarbeiter oft nicht erstrebenswert. Sie wünschen sich eine Kooperation, in der das Projekt eine gewisse (Entscheidungs-)Autonomie bewahrt. Um eine Gesprächskultur zwischen Projekt und Geldgeber zu etablieren, müssen angemessene Strukturen geschaffen werden (regelmäßige Termine, Verfahren für Entscheidungsfindung und Konfliktmanagement, Austausch von Informationen usw.). Eine gegenseitige Transparenz ist die Voraussetzung für eine gelungene Zusammenarbeit.

Aus Sicht der Praktiker ist die Entwicklung einer angenehmen Gesprächskultur auch mit anderen Kooperationspartnern wichtig. Dafür können diverse Methoden der Gruppengesprächsführung eingesetzt werden. Das Motto „Gemeinsam sind wir stark“ kann als Leitsatz dienen, um die Beteiligten anzuregen, sich mit gemeinsamen Interessen zu identifizieren und konkrete Formen der Kooperation zu überlegen. Treffen, auf denen sich die potenziellen Kooperationspartner präsentieren können („Dachveranstaltungen“), können dazu beitragen, fachliche Übereinstimmungen zu erzielen. Nicht selten werden Kooperationen von Geldgebern oder anderen (politischen) Entscheidungsträgern vorgeschrieben; aus der Erfahrung der Praktiker funktionieren solche „Zwangskooperationen“ jedoch unterschiedlich gut. Anregungen zur Kooperation „von außen“ können nützlich sein, aber die Gründe für die Kooperation müssen klar kommuniziert und der richtige Rahmen für die Zusammenarbeit geschaffen werden. Zwei weitere Punkte, die Probleme im Aufbau der Zusammenarbeit mit anderen Kooperationspartnern schaffen können, sind Scheinkooperationen und eine zu hohe Zahl an Beteiligten. Bei Scheinkooperationen ist keine Teilhabe an Entscheidungsprozessen vorgesehen, die $\mathrm{Ar}$ beitsbeziehung wird so jedoch nach außen dargestellt. Bei der Zahl an Beteiligten gilt das Sprichwort „Zu viele Köche verderben den Brei“: Eine zu hohe Zahl an Kooperationspartnern und/ oder Kooperationsvereinbarungen können die Wege länger machen und eine unnötige Bürokratie verursachen; dadurch wird die Qualität der Arbeit nicht verbessert, sondern die Arbeit wird verlangsamt und beeinträchtigt.

\section{Interessen}

Gemeinsame Interessen sind das Fundament jeder Zusammenarbeit, vor allem aber der partizipativen Zusammenarbeit, die einen hohen Grad an Abstimmung verlangt.

Die Interessen der Zielgruppe sind nicht immer einfach in Erfahrung zu bringen. Projektinteressen, die von den Förderinteressen des Geldgebers stark beeinflusst sind, werden oft mit den Interessen der Zielgruppe gleichgestellt. Es wird in der Regel von einer Bedürftigkeit der Zielgruppe ausgegangen, die im Rahmen von Antragsverfahren die Bereitstellung von Hilfeleistungen begründet, aber nicht unbedingt eine Basis für eine partizipative Zusammenarbeit mit der Zielgruppe bietet. Nicht selten definiert die Zielgruppe ihre Lebenslage anders und setzt andere Schwerpunkte in Bezug auf die Definition und Lösung von Gesundheitsproblemen. Wichtig ist, dass Wege außerhalb der Projekt- und Förderlogik gefunden werden, die Interessenslage der Zielgruppe in Erfahrung zu bringen. Möglich ist auch, dass die Zielgruppe nicht immer gemeinsame, sondern unterschiedliche Interessen hat, denn „Zielgruppen“ sind oft theoretische Konstrukte und keine lebensweltlichen Gruppierungen, die sich über ihre gemeinsamen Interessen verständigen.

Auch bei anderen Kooperationspartnern ist die Erkundung der Interessenlage von zentraler Bedeutung. Kleinere und größere Präferenzen, sowohl in Bezug auf Fachthemen als auch in Bezug 
auf Umgangsformen, sind beim Aufbau einer Zusammenarbeit zu beachten.

\section{Einigkeit in der eigenen Einrichtung}

Nicht selten kommt es in der Projektarbeit vor, dass eine Gruppe von Mitarbeitern eine Kooperation mit einer Zielgruppe oder anderen Kooperationspartnern anstrebt, ohne sich der $\mathrm{Zu}-$ stimmung ihrer Kollegen oder Vorgesetzen zu versichern. Durch die resultierende Uneinigkeit in der Einrichtung kann keine tragfähige Zusammenarbeit realisiert werden, weil die Interessen der Einrichtung nicht glaubwürdig und konsistent nach außen vertreten werden können. Aus diesem Grund gilt für viele Praktiker die Regel, zuerst Klarheit in der eigenen Einrichtung darüber zu schaffen, mit wem kooperiert werden soll, bevor konkrete Formen der Zusammenarbeit in die Wege geleitet werden.

\section{Fachlichkeit}

Eine weitere Voraussetzung für die erfolgreiche Zusammenarbeit eines Projekts mit allen Kooperationspartnern sind folgende Aspekte von Fachlichkeit:

- Klare Ziele und Standpunkte zu fachrelevanten Themen,

- Klarheit über die eigenen Grenzen in Verhandlungsgesprächen (Welche Kompromisse können wir als Einrichtung eingehen, welche nicht?),

- Kenntnisse über erfolgreiche, vergleichbare Projekte in anderen Kommunen,

- die Fähigkeit, über die eigene Arbeit nachvollziehbar zu kommunizieren, einschließlich der spezifischen Bedeutung der Arbeit für die Kommune (mit entsprechender Dokumentation, Fallbeispielen usw.),

- die Fähigkeit, die Belange der Zielgruppe (mit) zu vertreten,

- die Fähigkeit, sich an die Sprache von Politikern und anderen Entscheidungsträgern ebenso wie an die der Zielgruppe anzupassen und

- die Fähigkeit, Lobbyarbeit zu leisten.

\section{Aussicht}

Eine partizipative Zusammenarbeit zwischen Zielgruppe, Projekt, Geldgeber und anderen Akteuren zu realisieren, ist kein leichtes, jedoch ein zunehmend notwendiges und lohnendes Unterfangen, um die Qualität von gesundheitsfördernden und präventiven Maßnahmen zu steigern. Durch partizipative Herangehensweisen werden Praxiskompetenzen gestärkt und das Selbstbestimmungspotential von Menschen aus der Zielgruppe im Sinne von Empowerment aktiviert. Auf der Grundlage eines gemeinsamen Verständnisses von Partizipation als Entwicklungsprozess wird der Aufbau partizipativer Formen der Zusammenarbeit erleichtert - zum einen, weil die verschiedenen Ausprägungen der Partizipation konkretisiert werden, und zum anderen, weil die Möglichkeit zur Überprüfung der eigenen Zusammenarbeit nach dem aktuellen und erwünschten Stand der Partizipation besteht. Dadurch wird die Diskussion über Partizipation transparenter und für die Beteiligten handhabbar gemacht.

\section{Anmerkung}

Die Zusammenarbeit mit unseren Praxispartnern findet im Rahmen von zwei Forschungsprojekten statt: „Erfahrung nutzen Wissen vertiefen - Praxis verbessern: Partizipative Entwicklung der Qualitätssicherung und Evaluation in der Gesundheitsförderung für sozial Benachteiligte“ (Kooperationspartner Gesundheit Berlin e.V., gefördert vom BMBF, Förderkennzeichen: GFEL01062904/01EL0416) und „Strukturen zur Stärkung der Evaluation und Qualitätssicherung in der Primärprävention der Aidshilfen“ (Kooperationspartner: Deutsche AIDS-Hilfe e.V., gefördert durch die BZgA im Auftrag des BMG).

Interessenkonflikt: Für die Autoren besteht kein Interessenkonflikt.

\section{Literatur}

1 World Health Organization. Ottawa-Charta zur Gesundheitsförderung. Genf: WHO; 1986

2 Sachverständigenrat zur Begutachtung der Entwicklung im Gesundheitswesen (GC Fischer, G Glaeske, A Kuhlmey, R Rosenbrock, M Schrappe, FW Schwartz, PC Scriba, E Wille). Kooperation und Verantwortung. Voraussetzungen einer zielorientierten Gesundheitsversorgung. Bonn/Berlin: Bundesministerium für Gesundheit; 2007

3 Lehmann F. Kooperationsverbund zur Realisierung der Gesundheitsförderung bei sozial Benachteiligten in Deutschland. In: Richter M, Hurrelmann K, Hrsg. Gesundheitliche Ungleichheit. Grundlagen, Probleme, Konzepte. Wiesbaden: VS Verlag für Sozialwissenschaften; 2006; 423-438

4 Bär G, Buhtz M, Gerth H. Der Stadtteil als Ort von Gesundheitsförderung - Erfahrungen und Befunde aus stadtteilbezogenen Projekten. In: Rosenbrock R, Bellwinkel M, Schröer A, Hrsg. Primärprävention im Kontext sozialer Ungleichheit. Bremerhaven: Wirtschaftsverlag NW; 2004; 233-294

5 Wright MT. Partizipative Qualitätssicherung und Evaluation für Präventionsangebote in Settings. In: Rosenbrock R, Bellwinkel M, Schröer A, Hrsg. Primärprävention im Kontext sozialer Ungleichheit. Bremerhaven: Wirtschaftsverlag NW; 2004; 297-346

6 Israel BA, Schulz AJ, Parker EA et al. Review of community-based research: Assessing partnership approaches to improve public health. Annual Review of Public Health 1998; 19: 173-202

7 Minkler M, Wallerstein N Ed. Community Based Participatory Research for Health. Hoboken, New Jersey: John Wiley \& Sons; 2002

8 Arnstein S. A Ladder of Citizen Participation. Journal of the American Institute of Planners 1969 (4): 216-224

9 Hart R. Children's Participation. From Tokenism to Citzenship. Florence: UNICEF, International Child Development Center (Innocenti Research Center); 1992

10 Trojan A. 12-Stufen-Leiter der Bürgerbeteiligung (1988). In: BZgA Leitbegriffe der Gesundheitsförderung: Glossar zu Konzepten, Strategien und Methoden in der Gesundheitsförderung. 4. Auflage. Schwabenheim: Fachverlag Peter Sabo; 2003; 170

11 Wright MT, Block $M$, von Unger $H$. Stufen der Partizipation in der Gesundheitsförderung. InfoDienst für Gesundheitsförderung 2007; 7 (3): $4-5$

12 Galla J, Kopp U, Martinuzzi A et al. Programmakteursaufstellungen Erste Erfahrungen mit Systemaufstellungen in theoriebasierten Evaluationen. Zeitschrift für Evaluation 2008; 7 (1): 35-73

13 Wright MT, Lemmen $K$, Block $M$ et al. Setting up a peer-review process for community-based organizations: conflicts and challenges. Progress in Community Partnerships for Health 2008; 2 (2): 121-127

14 Deinet $U$, Szlapka M, Witte W. Qualität durch Dialog. Bausteine kommunaler Qualitäts- und Wirksamkeitsdialoge. Wiesbaden: VS Verlag für Sozialwissenschaften; 2008 